\title{
Integração entre o ensino de cálculo e o de pesquisa operacional
}

\author{
João Carlos C. B. Soares de Mello \\ Departamentos de Engenharia de Produção e de Metalurgia Industrial - Universidade Federal Fluminense \\ E-mail: jcsmello@producao.uff.br \\ Maria Helena C. Soares de Mello \\ Departamentos de Engenharia de Produção e de Ciência dos Materiais - Universidade Federal Fluminense \\ E-mail: gmamhel@vm.uff.br
}

\begin{abstract}
Resumo
Pretende-se mostrar neste trabalho como é possível ensinar, de forma integrada, as disciplinas de Cálculo Diferencial e de Pesquisa Operacional. Para tal são necessárias pequenas mudanças na forma como o Cálculo é tradicionalmente ensinado, mas grandes mudanças no ensino tradicional de Pesquisa Operacional. Com estas mudanças, Pesquisa Operacional pode cumprir o papel de disciplina integradora, fazendo a transição entre os ciclos básico e profissional no curso de Engenharia de Produção.
\end{abstract}

Palavras-chave

Cálculo Diferencial, Pesquisa Operacional, Ensino de Engenharia, Metodologia de Ensino Aprendizagem.

\section{How to teach Calculus and Operational Research in an integrated way}

\begin{abstract}
The aim of this paper is to show the possibilities of integration between the teaching of Differential Calculus and Operational Research. The integration is obtained with some small changes in the way that calculus is traditionally presented and some major changes in Operational Research classes. We show that, with the above-mentioned changes, Operational Research can play an important role in the transition between the first half and the last part of the Production Engineering courses.
\end{abstract}

\section{Key words}

Differential Calculus, Operational Research, Engineering Teaching, Learning Teaching Methodologies. 


\section{INTRODUĈ̣̃O}

A Pesquisa Operacional (PO) surgiu como disciplina formalizada durante a Segunda Guerra Mundial (1939 1945). O seu objetivo era fazer a integração entre estudos de equipes multidisciplinares com objetivo comum - aproveitar melhor os recursos ingleses e americanos na luta contra o III Reich. Tirar o máximo proveito de recursos escassos caracteriza os problemas de otimização (TAVARES et al., 1996). Na verdade, a palavra otimização foi, durante vários anos, quase que um sinônimo de Pesquisa Operacional.

Otimizar é uma atividade que ocupa grande parte da vida, pessoal ou profissional. Seja para maximizar a quantidade de bens e serviços adquiridos com o salário que se recebe, seja para obter o maior lucro possível para a empresa em que se trabalha, com os recursos disponíveis, ou reduzir ao máximo os custos operacionais.

Otimizar é também um conceito que faz parte dos programas de praticamente todos os cursos de Cálculo Diferencial e Integral. Embora seja um dos principais aspectos do Cálculo, o seu ensino é freqüentemente negligenciado pela suposta necessidade de resolver muitos exercícios de limites, derivação e integração. Os chamados problemas de Máximos e Mínimos são, em grande parte das turmas, apresentados de forma mecânica, sem explorar todo o potencial que eles apresentam para despertar o interesse do aluno e servir de motivação para o estudo de disciplinas posteriores.

técnicas do Cálculo Diferencial, também não é vista como matéria de matemática. A correção destes dois equívocos tornaria a PO uma excelente disciplina de ligação entre básico e profissional, verdadeiramente multidisciplinar. Este artigo pretende ser uma primeira abordagem para mostrar como, com pequenas mudanças de comportamento e metodologia, este potencial pode ser aproveitado.

O presente artigo é um detalhamento de trabalho apresentado por Soares de Mello \& Soares de Mello (2002b) e está organizado da seguinte forma: na próxima seção discorre-se sobre integração disciplinar de forma geral. As duas seções seguintes apresentam, respectivamente, considerações sobre o ensino de Cálculo Diferencial e Integral e de Pesquisa Operacional. Finalmente, são apresentadas as conclusões.

\section{INTEGRAC̣ÃO DISCIPLINAR}

A promulgação da Lei de Diretrizes e Bases da Educação Nacional - LDB em 1996 introduziu os vocábulos inter e multidisciplinaridade no cotidiano de quem esteja ligado à educação. No caso particular dos curso de Engenharia, as diretrizes curriculares (MEC, 2002) reforçam a necessidade de integração disciplinar. No entanto, o cotidiano mostra que esses conceitos estão restritos às conversas e intenções. A prática continua a ser o ensino segmentado ao longo dos cursos. Este é um problema especialmente grave nas várias habilitações dos cursos de Engenharia. De fato, em grande parte destes cursos, os conhecimentos básicos adquiridos nos primeiros períodos parecem dissociados e divorciados das aplicações utilizadas no ciclo profissional. É comum ouvirem-se comentários dos

O ensino mecanicista não é privilégio do Cálculo. É uma tentação a que sucumbem alguns dos professores de cursos introdutórios de Pesquisa Operacional. O caráter de multidisciplinaridade que estes cursos naturalmente deveriam ter é facilmente ignorado, e o ensino de Pesquisa Operacional é freqüentemente confundido com o de técnicas de Programação Linear. O estudo do algoritmo SIMPLEX tem tendência a dominar o curso, e pivotear torna-se uma palavra maldita para vários alunos.

Para os alunos de Engenharia de Produção, a Pesquisa Operacional é uma das primeiras disciplinas do chamado ciclo profissional. Se o seu ensino for reduzido a sucessivas operações algébricas, é natural que a sua aceitação como disciplina prática de Engenharia seja grandemente prejudicada. E, por não usar as arduamente aprendidas alunos com relação a este fenômeno. Reclamam, principalmente, que não percebem muito bem por que tiveram tantos cursos de Matemática (Cálculo, Álgebra e outros), se depois o máximo que utilizam de matemática são as quatro operações. Deve-se observar que alguns professores não tentam esclarecer a questão e alguns, ainda que de forma mais ou menos velada, concordam com o questionamento dos alunos.

Como professores das duas disciplinas, os autores deste trabalho identificaram estas dificuldades e vêm desenvolvendo algumas alterações metodológicas, tanto no Cálculo I quanto na disciplina Pesquisa Operacional Modelos Determinísticos que ministram na Universidade Federal Fluminense, com a intenção de reduzir este descompasso. 
Em várias Universidades, o ensino da Matemática ou da Física no Ciclo Básico é feito nos Institutos Básicos, por professores que desconhecem a seqüência do curso, embora muito competentes profissionalmente. Outra grande dificuldade aparece quando existe a distância física apreciável entre as unidades de ensino. Quando tal ocorre, o já escasso diálogo entres professores do Ciclo Básico e do Ciclo Profissional fica ainda mais prejudicado. Por outro lado, os Colegiados dos Cursos, que deveriam ser o fórum natural para promover entendimentos entre os professores das várias disciplinas, tratam, na maioria das vezes, apenas de problemas administrativos ou gerenciais, e deixam de lado, devido à existência de questões urgentes, ou ao excesso de trabalho, a discussão e a solução dos problemas didático-pedagógicos do curso.

A integração entre disciplinas básicas e profissionais é prejudicada pela tendência de ignorar os próprios defeitos e atribuir as responsabilidades pela deficiente formação dos alunos, no que diz respeito aos conteúdos, às habilidades e às atitudes, às etapas anteriores do aprendizado. Assim acontece com os professores do Ciclo Básico, que culpam a falta de conhecimentos que deveriam ter sido adquiridos no ensino médio e a pretensa deficiente seleção do vestibular pela excessiva reprovação nas suas matérias. Culpam ainda parte do desinteresse dos alunos em relação às matérias básicas de Matemática e Física pelo pouco uso que delas é feito em várias matérias do ciclo profissional.

Por outro lado, os professores do Ciclo Profissional atribuem a culpa pela falta de alunos e a notória evasão dos cursos de Engenharia ao excessivo rigor dos professores do Ciclo Básico, e à incapacidade destes de ensinarem o que realmente é importante para o futuro engenheiro.

Esta discussão, muitas vezes acirrada, é um entrave ao entendimento que poderia ajudar na solução de vários problemas. E, como é comum ocorrer em discussões, os argumentos usados pelos docentes das várias etapas do curso acabam por se mostrar corretos. Nem quem leciona para os primeiros períodos do curso costuma conhecer a conteúdo das matérias subseqüentes, ensinando o que acha que seja interessante, nem alguns professores do ciclo profissional se preocupam em fazer uso correto das ferramentas a que seus alunos foram apresentados nos primeiros períodos de estudos universitários. Fica assim aparentemente evidente a necessidade, conforme indicam as recém-aprovadas diretrizes curriculares para os cursos de Engenharia, das chamadas Disciplinas Integradoras, que têm a responsabilidade de agregar todo o conhecimento adquirido em um segmento do curso e utilizá-lo de forma eficiente.
De alguma maneira, em praticamente todos os cursos existem os Projetos Finais de Graduação, nos quais o aluno tem necessidade de utilizar muito do que aprendeu ao longo do seu curso. No entanto, é quase uma descoberta individual. E os "Projetos" têm se tornado, em muitos casos, muito mais uma dissertação ou monografia, com várias características das dissertações de conclusão de Mestrado, do que verdadeiramente um projeto de engenharia.

\section{prática continua a ser o ensino segmentado ao longo dos cursos.}

Em vez de criar disciplinas integradoras, pode-se fazer a integração disciplinar em algumas já existentes, que seriam assim transformadas em disciplinas integradoras sem deixarem de ser formadoras. E a Pesquisa Operacional, na Engenharia de Produção, é uma que se presta bastante bem para esta função.

\section{ENSINO DE CÁLCULO E ADAPTAC̄ÕES SUGERIDAS}

O ensino de Cálculo I tem passado por várias modificações mas, geralmente, sem perder a característica de ter a principal ênfase em exercícios repetitivos. As mudanças introduzidas, sempre com boas intenções, são decididas com base mais em idéias préconcebidas que em avaliações objetivas. Muitas vezes os resultados são o oposto do pretendido. Para o caso particular da Universidade Federal Fluminense, o histórico destas mudanças, suas motivações e conseqüências, pode ser encontrado em Soares de Mello et al. (2001, 2003) e Fernandes et al. (2001). Apesar dos dados referirem-se a um caso particular, a semelhança é muito grande com o que ocorreu em outras instituições.

A primeira grande mudança no ensino de Cálculo I ocorre no início da década de 1980, como resposta à constatação de que os alunos ingressavam no curso de engenharia com menor nível cognitivo. É uma reação extremamente simplista e imediatista: "se o aluno chega com menos conhecimento, e deve ao final do curso possuir todo o conhecimento necessário, é preciso tempo para ensinar o conhecimento que falta". Partindo de uma análise parcialmente correta, a solução incorreu em um grave erro. Foi proposto, e aceito, um aumento da carga horária de Cálculo I, de 60 horas para 90 horas semestrais. Ou seja, de 4 para 6 horas semanais. Com esse aumento de carga horária foi possível introduzir na programação vários conceitos elementares, tais como funções, polinômios, 
trigonometria, etc. Ressalte-se que esses assuntos eram, na maioria das vezes, abordados de forma operacional, sem preocupação com a fundamentação nem com o entendimento do conceito.

Contrariamente às expectativas, os índices de reprovação subiram bastante, tornando-se alarmantes. Pior, vinham acompanhados de um novo fenômeno: o alto índice de evasão.

O diagnóstico, provavelmente errado, é que o nível dos alunos que ingressavam continuava caindo. A situação de que o aluno, num só semestre, passava de conceitos simples de equação de retas e funções aos complexos domínios do "infinitamente pequeno", à derivação e suas aplicações, acabando em trabalhosos processos de integração de funções racionais, não levantava maiores reflexões. A reprovação era sempre atribuída à falta de estudo e foi a época em que proliferaram as listas de exercícios. Estas promoviam não o aprendizado, mas o adestramento em resolver problemas repetitivos. cais em sentido oposto. Na UFF esta mudança ocorreu em 1994, com a redução da carga horária e a mudança de enfoque da matéria, que passou a ser mais conceitual. A experiência, embora de resultados animadores, foi parcialmente interrompida com a ênfase no uso de recurso computacionais no lugar do trabalho no entendimento conceitual.

Há que se ter cuidado com a utilização indiscriminada dos recursos tecnológicos. Em alguns casos, uma experiência malsucedida pode comprometer vários anos de avanços (SOARES DE MELLO et al., 2002). Na UFF, a utilização do programa Maple para o ensino de Cálculo, de forma malfeita, além de ter sido ineficiente, reduziu as possibilidades de se experimentar outras novas tecnologias. Por outro lado, atualmente tem sido fundamental recuperar pré-conceitos para o Cálculo no Cálculo I (NASCIMENTO, 2000, 2002), e o aprendizado de uma nova técnica pode ser um impedimento para o aprendizado dos conceitos necessários. É possível que seja muito mais útil só utilizar softwares para o ensino do Cálculo após os alunos dominarem os conceitos, isto é, cerca de dois períodos mais tarde, em outras matérias de matemática, como Equações Diferenciais.

A aridez do Cálculo é um dos obstáculos ao seu aprendizado. Além de introduzir notas históricas, o uso de linguagem e exemplos que virão a fa-

Com os atuais recursos tecnológicos não parece ter validade fazer com que os alunos repitam exercícios iguais com os chamados "malabarismos algébricos". As provas já não deveriam avaliar apenas as habilidades conquistadas pela repetição, o que tornaria desnecessárias as listas de exercícios.

O tipo de ensino descrito acima tem por conseqüência a não retenção de conhecimentos. Tal fato começou a ser notado quando se verificou que no Cálculo II a reprovação também era grande. Aparentemente isto era uma contradição já que, segundo se acreditava, o Cálculo I só aprovava quem sabia muito. Acresce que é notória a existência de várias gerações de alunos que, mesmo tendo sido aprovados, ganharam aversão tão grande à matemática de um modo geral que há, em muitos casos, quase um bloqueio psicológico quando se tenta, em disciplinas do Ciclo Profissional, utilizar-se dos conteúdos que os alunos deveriam dominar. Chegam a ser ridículos os malabarismos que alguns docentes acabam por fazer para evitar o uso de conceitos de Cálculo. Muitas vezes até pedem desculpas por ter que colocar um símbolo de integral no quadro. Isto sem falar em álgebra de matrizes, operadores lineares, modelos matemáticos, etc.

Assim, ficou patente a necessidade de mudanças radi- zer parte do conteúdo do ciclo profissional pode contribuir para aumentar a motivação dos alunos e, portanto, melhorar o aprendizado. Um dos tópicos que melhor se presta a quebrar a aridez do Cálculo, é a teoria de máximos e mínimos.

De fato, o Cálculo I é o primeiro contato formal com a otimização. Não se deve negligenciar esta grande utilização do Cálculo. Ao se estudar as teorias de máximos e mínimos de funções, particularmente de funções de uma variável, é importante não se fixar somente no estudo de variações de funções, com o objetivo de serem traçados seus gráficos. Quase todas as calculadoras gráficas modernas hoje em dia realizam essa tarefa, com bastante precisão. Também os problemas de solução automática, ou cuja aplicabilidade não seja evidente, devem ser evitados. Exemplos destes problemas, encontrados nos textos padrão de Cálculo (SWOKOWSKI, 1995) são: "quais as dimensões do retângulo de maior área possível com perímetro dado", ou "qual o melhor ponto de desembarque numa praia, dado que o pescador rema a uma velocidade constante $v$ e corre a uma velocidade constante $v^{\prime}$, para que ele chegue o mais rapidamente possível em sua casa que fica à beira-mar, conhecida a posição 
inicial do barco em relação à casa".

O Cálculo I fica restrito a problemas com apenas uma variável independente. Aliás, esse nome não ajuda à integração com Pesquisa Operacional ou outras matérias do ciclo profissional. Posteriormente será utilizado em Pesquisa Operacional o nome "variável de decisão", enquanto que os engenheiros mecânicos usarão a designação "variável de projeto".

A introdução precoce das linguagens verdadeiramente utilizadas, com os termos que são aplicados na vida profissional, economiza muito tempo no futuro, e ajuda a fazer a ligação entre as disciplinas. Além da denominação atribuída a variáveis, outro exemplo de descompasso entre denominações ocorre nos cursos de Eng. Elétrica e Telecomunicações. Em um mesmo período letivo, o aluno utiliza a letra $i$ como representante de intensidade de corrente elétrica, nas disciplinas de Circuitos Elétricos, como $\sqrt{-1}$ em Matemática e taxa de juros em Matemática Financeira.

Não é só na denominação que se pode melhorar a integração entre disciplinas. A própria apresentação dos problemas estudados pode sofrer pequenas adaptações, potencialmente úteis. Assim, é possível já em Cálculo I tratar da modelagem de problemas, ao invés de chamálos simplesmente de problemas de otimização ou, pior ainda, problemas de máximos e mínimos. Tenta-se, no exercício desta modelagem, identificar sempre o objetivo, determinar a função objetivo, apresentar as restrições e inseri-las no problema. Mostra-se claramente que um modelo é uma representação da realidade, em alguns casos, admitindo que se cometem erros. Deve-se deixar claro que as equações não são dogmas aos quais a realidade deve se submeter.

O já mencionado problema da área do retângulo pode ser assim formulado: "Um criador de galinhas deseja fazer um cercado na forma de um retângulo para seus animais, de maneira que eles tenham a maior possibilidade de movimentação. Ele deseja utilizar toda uma cerca que dispõe, medindo $m$ metros, e precisa definir quais as dimensões deste cercado."

Pode-se definir o objetivo - construir o galinheiro, na forma retangular, com a maior área possível; a função objetivo - sendo $l$ e $b$ as dimensões (largura e comprimento do cercado) maximizar $\mathrm{S}=b . l$; e as restrições $-p$ sendo o perímetro, $p=2 .(l+b)=m ; l, b \geq 0$.

Este problema simples é aproveitável para apresentar conceitos que serão extremamente relevantes posteriormente: basta formular o problema em que é necessário cercar uma área prédefinida e deve-se utilizar a menor quantidade possível de cerca. A solução deste problema conduz ao mesmo quadrado obtido anteriormente. $\mathrm{Ou}$ seja, dois problemas aparentemente diferentes, um de maximizar, outro de minimizar mostram ter semelhanças estruturais e conduzem ao mesmo resultado. Está, de forma ainda intuitiva, introduzido o conceito de dualidade, que surgirá formalmente quando o aluno estudar Pesquisa Operacional.

Pode-se ainda aproveitar os conceitos básicos de juntos abertos e fechados, já pensando na construção da região viável. E, tanto em Cálculo I como em Cálculo II, pode-se - e deve-se - enfatizar que, em muitas das situações, quando se tem uma função definida num intervalo fechado, nem sempre o ponto de máximo ou de mínimo é aquele em que a derivada da função é zero.

No Cálculo II, aumentam-se as possibilidades, podendo-se trabalhar com várias variáveis, possibilitando a incorporação das restrições ao problema por meio dos multiplicadores de Lagrange. Muitas vezes o aluno é apenas treinado a trabalhar com este tipo de abordagem, automaticamente. Popularmente, ele "deriva e iguala a zero". O fundamental estudo da fronteira raramente é bem enfatizado, o que pode levar a erros posteriores. Alguns textos mais completos (BORTOLOSSI, 2002) chegam a apresentar as condições de Karush-Kuhn-Tucker, mas de forma apenas algébrica, sem explorar o potencial da sua interpretação geométrica para o entendimento dos problemas de otimização.

A correta e completa interpretação do teorema de Weirstrass, junto com a técnica de análise da fronteira, permitiria apresentar o método gráfico usado na solução de Problemas de Programação Linear de duas dimensões, ainda no Cálculo II. Bastaria apresentar exemplos em que a função é linear (e portanto não tem derivadas parciais nulas em nenhum ponto). Se as restrições forem igualmente lineares, já são introduzidos alguns dos problemas clássicos de Programação Linear.

\section{ENSINO DE PO E POSSÍVEIS ADAPTAC̣ÕES}

Da mesma maneira, o ensino de PO também anda necessitado de reformulações. Em muitas situações mantêm-se os paradigmas de antes da computação algébrica, e 
de microcomputadores disseminados. Insiste-se em que o ensino de PO limita-se a problemas de programação linear, com ênfase quase que total no algoritmo SIMPLEX. Neste caso, focaliza-se a solução prioritariamente no uso de sucessivos pivoteamentos manuais e dezenas de somas de frações. O problema é agravado pelo fato de os livros tradicionais (MACULAN FILHO e PEREIRA, 1980; BREGALDA e BORNSTEIN, 1981), ainda largamente usados, apresentarem nos primeiros capítulos uma extensa revisão dos conceitos de Álgebra Linear. É evidente que esta abordagem desestimula os alunos. Alguns textos mais modernos iniciam com o histórico da PO ou com a apresentação de técnicas e exemplos de modelagem (CAIXETA FILHO, 2000; GOLDBARG e LUNA, 2000). Mas, mesmo estes, acabam por insistir na resolução manual do SIMPLEX e seu fundamento algébrico. caso em Programação Linear em que realmente uma derivada é zero, já que ao longo da face a função objetivo permanece constante.

Esta abordagem evita o descompasso entre disciplinas, já que os conceitos de Cálculo são usados em Pesquisa Operacional. Se, somada a isto, for dada uma grande ênfase à modelagem de problemas, o caráter aplicado da PO é realçado. Ou seja, é feita a ligação entre os conceitos aprendidos no Ciclo Básico e a aplicação aos problemas de Engenharia. Para esta abordagem, não basta mostrar os problemas clássicos. É conveniente estimular os alunos a procurarem por si mesmos soluções para problemas como corte de chapas, planejamento da produção, dimensionamento de frotas, e outros. Mais importante ainda é fazer com que os alunos formulem problemas novos, tirados de situações reais. Só assim o aspecto prático da PO pode ser realçado.

Neste momento, a larga utilização de softwares como o LINDO ou LINGO, ou outros, é de grande valia, já que os alunos, que detiveram o conceito, podem compreender que mais importante do que a sua habilidade em fazer contas com fração é a sua habilidade em modelar bem um pro-

Além do sentimento de inutilidade em realizar operações repetitivas, mais próprias de um computador, outra situação aflige o aluno: ele julga ter aprendido que a otimização resume-se a igualar a zero algumas derivadas. No máximo, admite fazer algum teste com derivadas de segunda ordem. E, ao longo de todo o aprendizado de Programação Linear, não vê uma única derivada. É inevitável a sensação de que algum dos professores o está enganando.

E, no entanto, é fácil evitar esta situação. Basta, no lugar de complicadas operações algébricas, mostrar que não pode haver extremos no interior da região viável, já que a linearidade da função objetivo - variável dependente - impede que as derivadas se anulem. Raciocínio semelhante aplicado à fronteira da região conduz à procura de extremos nos vértices. É então mostrado ao aluno que ele não irá calcular nenhuma derivada, mas que precisa usar os conceitos de cálculo para chegar a essa conclusão. Isso o leva a concluir que, nos casos em que a função objetivo não seja linear, ele não deverá ignorar o fato de que a solução pode estar no interior da região viável ou em qualquer outro ponto da fronteira. Ou seja, um aprendizado de Programação Linear com base em conceitos de Cálculo pode facilitar o futuro aprendizado de programação não-linear.

No estudo de problemas especiais pode ainda ser mostrado que o caso de múltiplas soluções ótimas é o único blema e compreender e interpretar os resultados e todas as possibilidades que o programa pode lhes oferecer.

\section{CONCLUSÕES}

As várias possibilidades de mudanças no ensino de Cálculo e de Pesquisa Operacional mostram que a PO é uma disciplina que se presta muito bem ao papel de disciplina integradora da matemática aprendida no ciclo básico e de transição para solução de problemas, verdadeiramente multidisciplinar. Deve, na progressão curricular, estar colocada logo após o fim do ciclo básico, de forma que possa agregar todo os conceitos recémapreendidos. No entanto, é preciso que ao longo do curso outras disciplinas sejam integradoras. E que nestas disciplinas se utilizem os conceitos básicos de um primeiro curso de PO, em conjunto com as demais disciplinas cursadas. Também é fundamental cuidar para que não se cometa em relação à $\mathrm{PO}$ o mesmo erro que é comum os professores de $\mathrm{PO}$ cometerem em relação ao Cálculo e à Álgebra, ou seja, o de ignorar que os alunos já devem dominar este conteúdo. Cada vez mais é importante, nos cursos em regime de créditos principalmente, que se faça a correlação entre os conhecimentos apreendidos e as habilidades desenvolvidas nas disciplinas, evidenciando ao aluno que o seu curso não é um conjunto de discipli- 
nas, mas que elas têm uma razão de ser. As metodologias apresentadas neste trabalho não pretendem ser as únicas capazes de tornar o curso de Pesquisa Operacional mais interessante. Outras experiências têm sido feitas e podem ser encontradas em, por exemplo, Coutinho et al. (2003), que desenvolvem um curso de Pesquisa Operacional por intermédio do projeto de um flutuador.
Deve-se também enfatizar que as mudanças sugeridas no ensino de Cálculo não obrigam a turmas de Cálculo específicas para Engenharia de Produção. Na verdade, as mudanças aqui sugeridas, junto com outras que integrem o Cálculo com diversas matérias do profissional, tornam esta matéria mais atraente para alunos de todas as engenharias.

\section{Edição especial início $07 / 03 / 2003$ fim 25/08/2003}

\section{- Bibliografia}

BORTOLOSSI, H. J. Cálculo Diferencial a Vária Variáveis. Uma introdução à Teoria da Otimização. Rio de Janeiro : Editora PUC-Rio, 2002.

BREGALDA, P.A.O., BORNSTEIN, C. Introdução à Programação Linear. Rio de Janeiro : Editora Campus, 1981.

CAIXETA FILHO, J. V. Pesquisa Operacional. Técnicas de Otimização Aplicadas a Sistemas Agroindustriais. São Paulo : Editora Atlas, 2000.

COUTINHO, H. J. S., KERN, V. M., WICHROWSKY, H. C. Estratégia de Ensino para Inicialização na Modelagem de Problemas de Programação Linear. In: Anais do XXXI COBENGE - Congresso Brasileiro de Ensino de Engenharia, Rio de Janeiro, RJ, 2003.
FERNANDES, A. J. S., SOARES DE MELLO, M. H. C, SOARES DE MELLO, J. C. C. B. Ensino de Cálculo I: evolução na UFF. In: Anais do VII EEE - Encontro de Educação em Engenharia, Petrópolis \& Iguaba Grande, RJ, 2001.

GOLDBARG, M.C., LUNA, H.P.L. Otimização Combinatória e Programação Linear, Modelos e Algoritmos. Rio de Janeiro : Editora Campus, 2000.

MACULAN FILHO, N., PEREIRA, M. V. F. Programação Linear. São Paulo : Editora Atlas, 1980.

MEC. Diretrizes Curriculares Nacionais dos Cursos de Engenharia. Parecer CNE/ CES $1.362 / 2001$ de $12 / 12 / 2001$. Diário Oficial da União, 25/02/2002.
NASCIMENTO, J.L. A recuperação dos pré-conceitos do Cálculo. In: Anais do XXVIII COBENGE - Congresso Brasileiro de Ensino de Engenharia, Ouro Preto, MG, 2000

NASCIMENTO, J.L. Matemática: conceitos e pré-conceitos. In: PINTO, D.P., NASCIMENTO, J.L. Educação em Engenharia - Metodologia. pp 247 - 295. São Paulo : Editora Mackenzie, 2002.

SOARES DE MELLO, J. C. C. B., LINS, M. P. E., SOARES DE MELLO, M. H. C., GOMES, E. G. Evaluating the Performance of Calculus Classes Using Operational Research Tools. European Journal of Engineering Education, v. 27, n. 2, pp 209-218, 2002

SOARES DE MELLO, J. C. C. B., SOARES DE MELLO, M. H. C. Integração entre o ensino de cálculo e o de pesquisa operacional. In: Anais do XXII ENEGEP Encontro Nacional de Engenharia de Produção, Curitiba, PR, 2002b.
SOARES DE MELLO, J.C.C.B., SOARES DE MELLO, M.H.C., FERNANDES, A.J.S. Mudanças no ensino de Cálculo I: histórico e perspectivas. In: Anais do XXIX COBENGE - Congresso Brasileiro de Ensino de Engenharia, Porto Alegre, RS, 2001.

SOARES DE MELLO, M. H. C., SOARES DE MELLO, J. C. C. B., FERNANDES, A. J. S. História e reflexão crítica do ensino de Cálculo I: estudo de caso na Universidade Federal Fluminense (UFF). In: Anais do XXVI CNMAC - Congresso Nacional de Matemática Aplicada e Computacional, São José do Rio Preto, SP, 2003

SWOKOWSKI, E. W. Cálculo com Geometria Analítica. Rio de Janeiro : Makron Books do Brasil Editora Ltda., 1995.

TAVARES, L. V., OLIVEIRA, R. C., THEMIDO, I. H., CORREIA, F. N. Investigação Operacional. Lisboa : Mc Graw Hill, 1996 